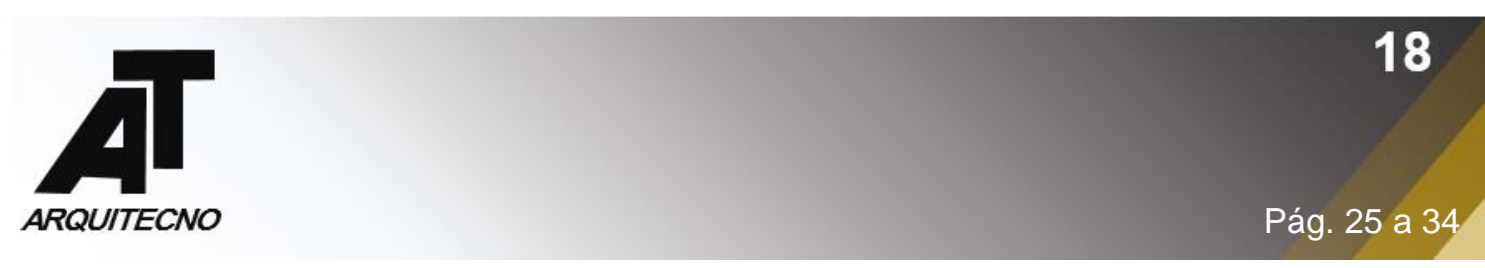

\title{
Arquitectura y confiabilidad estructural
}

\section{Architecture and structural confiability}

Esp. Ing. Silvina Inés Prados, Ing. Anabella Loriana Cardellino, Arq. Alexandra Abigail Vallejos, Arq. Madeleine Ledezma Orozco

\begin{abstract}
Estructuras IIB, Taller de investigación de diseño estructural, Facultad de Arquitectura,
\end{abstract} Urbanismo y Diseño, Universidad Nacional de Córdoba, Córdoba, Argentina.

estructuras2b@faud.unc.edu.ar

\section{RESUMEN}

El siguiente trabajo es el resultado de la investigación llevada a cabo por las integrantes del proyecto "regularidad estructural como recurso para un diseño sostenible" de la Universidad Nacional de Córdoba. El objetivo perseguido es "visibilizar en los diseños estructurales la incidencia de la regularidad estructural en el desarrollo de proyectos arquitectónicos sostenibles". Para ello, con la ayuda de programas de cálculo estructural, modelamos edificios con diferentes grados de irregularidad y evaluamos cuantitativamente el consumo de material requerido para cada una de ellas. Los resultados obtenidos hasta el momento son satisfactorios donde se llegaron a reducciones de hasta un 15\% del volumen de hormigón armado en estructuras regulares y por lo tanto confiables estructuralmente. Como formadoras y profesionales estamos convencidas que estos avances deben darse a conocer y transferirse a la formación de grado del futuro arquitecto para constituirse en criterios de diseño que garanticen arquitecturas seguras, duraderas y sostenibles.

\section{ABSTRACT}

The following work is the result of the research carried out by the members of the project "structural regularity as a resource for sustainable design" of the National University of Córdoba. The objective pursued is "to make visible in structural designs the incidence of structural regularity in the development of sustainable architectural projects". To do this, with the help of structural calculation programs, we model buildings with different degrees of irregularity and quantitatively evaluate the material consumption required for each of them. The results obtained so far are satisfactory where reductions of up to $15 \%$ in the volume of reinforced concrete were reached in regular structures and therefore structurally reliable. As trainers and professionals, we are convinced that these advances must be made known and transferred to the undergraduate training of the future architect to become design criteria that guarantee safe, durable and sustainable architectures.

PALABRAS CLAVES: Regularidad estructural, Diseño sostenible, Hormigón armado

KEY WORDS: Structural regularity, Sustainable design, Reinforced concrete

FECHA DE RECEPCIÓN: 25/8/2021| FECHA DE ACEPTACIÓN: 8/11/2021

DOI: http://dx.doi.org/10.30972/arq.0185667 


\section{INTRODUCCIÓN}

Los recientes acontecimientos sucedidos en la ciudad de Miami durante el mes de julio de 2021 dan muestras de que la confiabilidad estructural debiera ser una condición indiscutible que debe cumplir todo proyecto de arquitectura. Los terremotos, los asentamientos diferenciales 0 descalces de las fundaciones, las inestabilidades locales de los elementos estructurales, los ineficientes e inexistentes mantenimientos, la degradación de los materiales debido a las agresiones ambientales, etc., no debieran ser fenómenos ajenos a las decisiones de proyecto sino más bien, nutrirlas. Existen sobradas muestras a través de la historia que aquellos proyectos con comportamientos previsibles o confiables, tanto para solicitaciones estáticas como dinámicas, responden de manera más dúctil a las incertidumbres derivadas de la etapa constructiva, el uso durante la vida útil de la construcción y hasta para su sobrevida o reciclaje.

Por otro lado, es bien sabido que la industria de la construcción es una de las grandes consumidoras de energía en todo el mundo durante el proceso de elaboración de los materiales, edificación y sobre todo para la etapa de uso de los edificios (Prados y Cardellino, 2020, p.119). A esto se suma que los materiales más utilizados y adecuados para algunas tipologías, usan materias primas no renovables con procesos de producción que requieren del consumo de abundante agua y emiten grandes cantidades de dióxido de carbono al ambiente, no aportando, por lo tanto, a un desarrollo sostenible.

Con el fin de accionar con medidas correctoras ante esta situación, la mayoría de los sistemas de certificación actuales, que regulan el grado de sostenibilidad de las edificaciones, para las etapas de diseño y construcción, priorizan aspectos ambientales como la disminución del consumo de agua y de energía, las acciones llevadas a cabo para controlar las emisiones de dióxido de carbono en los procesos empleados y la naturaleza de los materiales utilizados, pero dejan de lado la importancia de un buen diseño estructural. Esto podría traducirse en importantes disminuciones de los recursos materiales, pero también en construcciones resistentes y seguras con períodos de vida útil considerables, o sea en un buen uso de esos materiales (Prados y Cardellino, 2020, p.133).

Según la Real Academia Española, se entiende por eficiencia la "capacidad para realizar o cumplir adecuadamente una función" y esto estuvo asociado a un buen diseño estructural a través de la historia de la arquitectura, relacionando solo al consumo de materiales para los diferentes elementos estructurales, por ejemplo, acero u hormigón, con su capacidad de resistir satisfactoriamente las solicitaciones que los demandaban. En la actualidad el concepto de eficiencia estructural debiera renovarse en base a nuevos estándares culturales y tecnológicos e incorporar además el nivel de seguridad o confiabilidad con el que estos elementos en esas condiciones cumplen dicha función y si este es aceptable por la sociedad al verificar lo especificado en las reglamentaciones vigentes.

El reglamento INPRES-CIRSOC 103 en su versión del año 2016, incorpora la variable de regularidad estructural, tanto en planta como en altura, para la definición de los métodos de diseño en construcciones de hormigón armado en zona sísmica. De su lectura se deduce que un buen diseño corresponde al de una estructura regular que, al minimizar los efectos torsionales, tiene un comportamiento previsible frente a la acción sísmica proponiendo para ellas métodos de análisis sencillos y coeficientes de seguridad que contemplan menores incertidumbres. Mientras que, una estructura con un grado de irregularidad elevado, debido a su gran vulnerabilidad y a la ineficiencia de los métodos de diseño actuales en la determinación del real comportamiento de las mismas, las considera inadmisibles en zonas geográficas de elevado riesgo sísmico (Prados y Cardellino, 2020, p.119).

Existen innumerables recursos para disminuir las acciones equivalentes que producen los terremotos sobre las construcciones como son el uso de aisladores y disipadores sísmicos, aunque estos no son tan económicos ni accesibles en nuestro país y, por lo tanto, poco 
frecuentes en el diseño de algunas tipologías como es el caso de la vivienda colectiva. Es así que, al igual que un acondicionamiento térmico pasivo es considerado sostenible, un diseño estructural regular constituye un recurso pasivo y por lo tanto sustentable para la arquitectura.

El proyecto de investigación que presentamos a continuación pertenece al Taller de Investigación de Diseño Estructural de la Facultad de Arquitectura, Urbanismo y Diseño de la Universidad Nacional de Córdoba y es llevado a cabo por un equipo interdisciplinario, integrado por ingenieras y arquitectas, que intentan demostrar que un diseño estructural regular es más eficiente al demandar no solo menos consumo de material sino poseer una mayor confiabilidad ya que su comportamiento es fácilmente previsible por los diseñadores pudiendo estos evaluar decisiones proyectuales de forma acertada.

\section{METODOLOGÍA}

El proyecto del cual formamos parte lleva por título "regularidad estructural como recurso para un diseño sostenible" y tiene por objetivo "visibilizar en los diseños estructurales la incidencia de la regularidad estructural en el desarrollo de proyectos arquitectónicos sostenibles".

Para llevarlo a cabo, se dividió en 4 etapas (Tabla 1):

- Etapa 1: Relevamiento y estudio bibliográfico para la construcción del marco teórico de la problemática planteada.

- Etapa 2: Búsqueda y selección de los casos de estudio. Los parámetros a tener en cuenta para la realización de esta etapa fueron: debe tratarse de un edificio en altura construido, con un diseño estructural irregular, ubicado en una zona de baja sismicidad. Posteriormente se realizó el modelado de la estructura con un programa de elementos finitos.

- Etapa 3: Optimización del mecanismo estructural existente hasta garantizar una regularidad estructural baja. Se procedió al análisis y verificación de la propuesta aplicando, según se indica en el reglamento INPRES-CIRSOC 103, el método estático equivalente. Esto permite transferir las conclusiones a la formación de grado del futuro arquitecto.

- Etapa 4: Constatación con la hipótesis. Elaboración de las conclusiones. Divulgación de los resultados. Elaboración de material didáctico.

Esta etapa es la que se está desarrollando actualmente donde las primeras conclusiones serán compartidas en esta publicación.

Tabla 1: Etapas y su desarrollo en el tiempo

\begin{tabular}{|c|c|c|c|c|c|c|}
\hline \multirow{2}{*}{ Actividades } & \multicolumn{3}{|c|}{2020} & \multicolumn{3}{|c|}{2021} \\
\hline & $\begin{array}{l}1 \stackrel{\circ}{\circ} \\
\text { trim }\end{array}$ & $\begin{array}{l}2 \stackrel{\circ}{0} \\
\text { trim }\end{array}$ & $\begin{array}{l}3 \stackrel{\circ}{ } \\
\text { trim }\end{array}$ & $\begin{array}{l}1 \stackrel{0}{0} \\
\text { trim }\end{array}$ & $\begin{array}{l}2 \stackrel{\circ}{0} \\
\text { trim }\end{array}$ & $\begin{array}{l}\stackrel{\circ}{\circ} \\
\text { trim }\end{array}$ \\
\hline $\begin{array}{l}\text { Estudio bibliográfico y } \\
\text { elaboración del marco teórico }\end{array}$ & & & & & & \\
\hline $\begin{array}{l}\text { Búsqueda y selección de los } \\
\text { casos de estudio y primera } \\
\text { etapa de modelado }\end{array}$ & & & & & & \\
\hline $\begin{array}{l}\text { Optimización de la } \\
\text { configuración estructural de } \\
\text { los proyectos analizados y } \\
\text { segunda etapa de modelado }\end{array}$ & & & & & & \\
\hline
\end{tabular}






DESARROLLO

Fuente: elaboración propia

\section{Etapa 1}

Esta primera etapa fue fundamental para el desarrollo de las siguientes ya que el incipiente grupo de trabajo constituye un equipo en formación. Las actividades llevadas a cabo fueron:

- Capacitación del equipo de trabajo. En esta etapa fue importante la capacitación referente al uso de los programas de cálculo que se utilizarían como herramientas de diseño así mismo como el estudio pormenorizado de las reglamentaciones vigentes en nuestro país y en la región para tomar de referencia.

- Se profundizó en el estudio bibliográfico sobre el tema de estudio y se procedió a la búsqueda de nuevos antecedentes referenciales.

- Se participó de seminarios, charlas y cursos de formación específica en la disciplina.

- Se procedió a la elaboración y publicación del marco teórico.

\section{Etapa 2}

A continuación, se comenzó con la búsqueda de los potenciales casos de estudio a analizar durante el proyecto para permitirnos encontrar respuestas a nuestros interrogantes.

Con este objetivo, la búsqueda estaba orientada a edificios de hormigón armado, irregulares, de mediana altura (hasta 15 niveles), cuyo destino sea la vivienda y se encuentren ubicados en zonas de baja sismicidad.

De todos los casos seleccionados se eligió el edificio Pueyrredón 1101 diseñado por el estudio de Pablo Gagliardo y ubicado en la ciudad de Rosario.

El edificio se construyó entre los años 2014 y 2017, posee un nivel de subsuelo y 14 niveles sobre nivel de vereda teniendo una altura total de 42 metros.

Gentilmente el estudio de arquitectura nos proporcionó toda la información del proyecto lo cual facilitó la etapa de relevamiento y modelado de la estructura.

Cabe destacar que, para el análisis requerido por el proyecto, fue menester analizar el edificio ubicado en una zona de sismicidad media (zona sísmica 2), modificando sus condicionantes de proyecto originales $y$, por lo tanto, las conclusiones obtenidas nada tienen que ver con las condiciones reales del edificio construido.

Desde el punto de vista programático la obra está compuesta por unidades flexibles abiertas hacia los frentes norte y oeste con balcones, permitiendo la expansión del espacio interior hacia el exterior. Se agrupan 3 departamentos con tipologías diferentes en cada nivel, disponibles para vivienda u oficinas. Las zonas compartidas como el comedor de usos múltiples, la piscina y terraza se encuentran en los últimos dos niveles. Se concibe un ingreso de doble altura como una continuidad del espacio público hacia el interior del edificio, que junto con la abundante vegetación de la vereda y un gran bicicletero recrean una plaza interna. En la planta baja se ubican además locales comerciales y oficinas (Figura 1). 

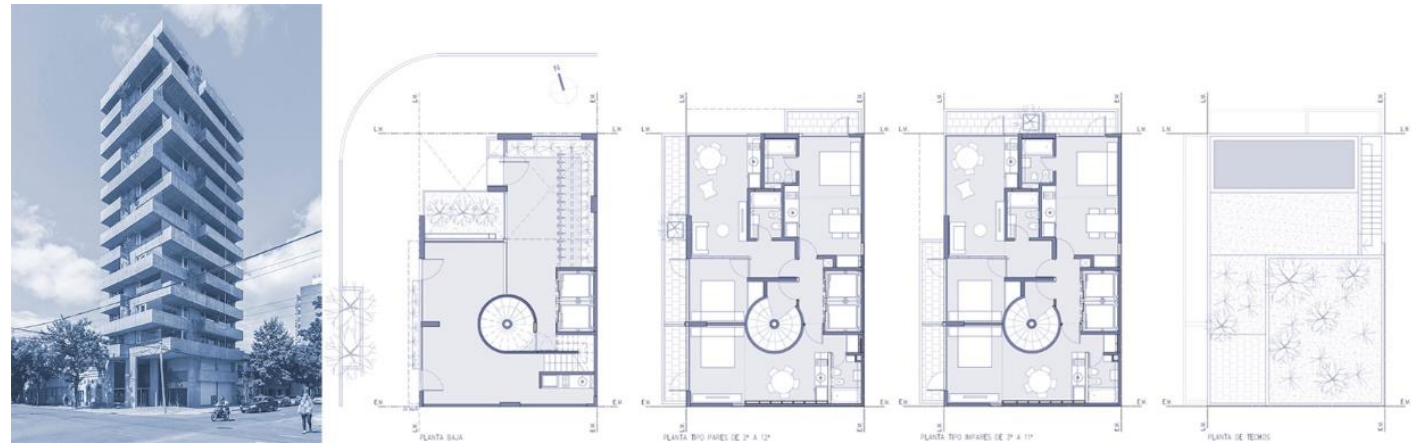

Figura 1: Imagen exterior. Planta baja, planta tipo par e impar y planta terraza. Fuente: elaboración propia

La estructura de hormigón armado a la vista es el lenguaje expresivo del proyecto que es realzado con las marcas del entablonado del encofrado. Conformado por tabiques, columnas y vigas en voladizo, que en fachada se intercalan y materializan los balcones, generando un juego de dobles alturas que favorecen una mayor apertura de las unidades hacia los frentes y logran una interesante calidad espacial.

Los planos horizontales están compuestos por losas macizas hacia los frentes y losas nervuradas hacia el interior del edificio (Figura 2).



Figura 2: Plantas de estructuras, par e impar y despiece por nivel de la estructura. Fuente: elaboración propia 


\section{Etapa 3}

En esta instancia se procedió a la optimización de la estructura original hasta llegar a valores de regularidad estructural aceptables según la reglamentación vigente en nuestro país.

Como primera alternativa se analizó el caso de sustituir los tabiques de hormigón armado en coincidencia con las medianeras por algún tipo de envolvente que permita el comportamiento de pórticos en toda la altura del edificio coincidente con los pórticos existentes en planta baja con el fin de evitar la gran reducción de rigidez que existe en la transición con los niveles inferiores, lo que produciría una falla en el edificio conforme con las de piso blando (Figura 3).

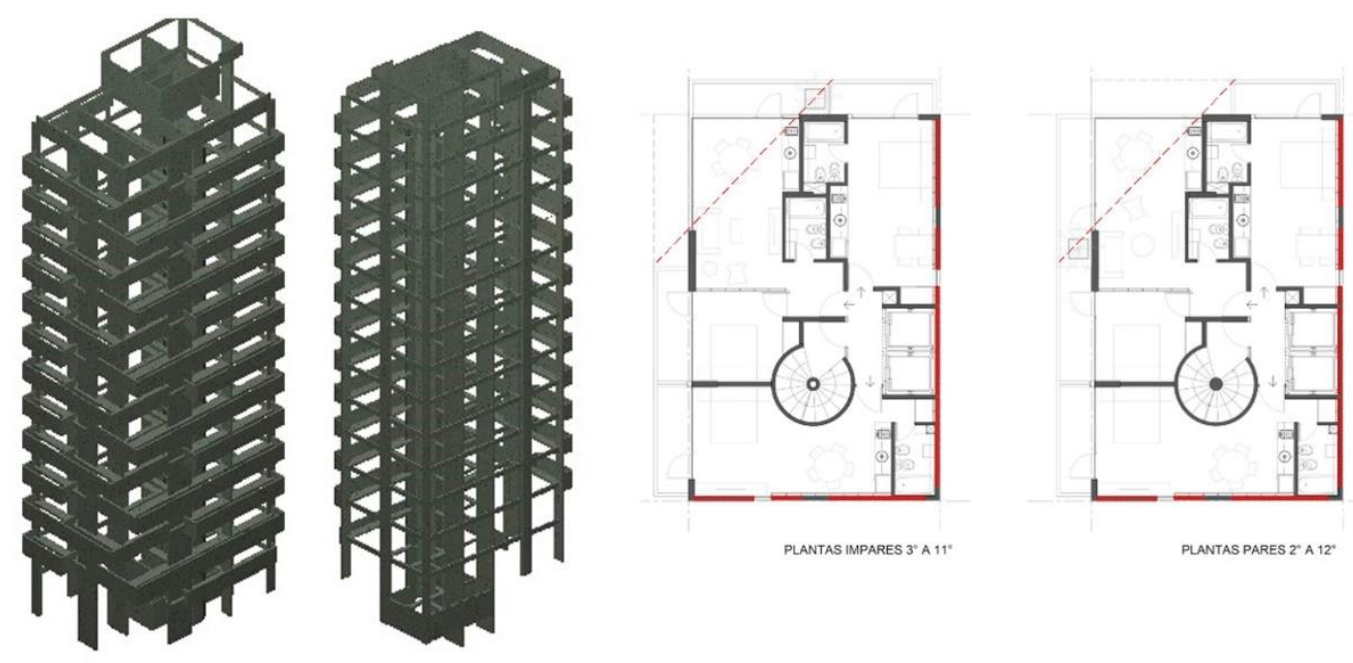

Figura 3. Modelado y plantas con la propuesta estructural

De este análisis, si bien se logró eliminar el factor determinante de la irregularidad detectada en el proyecto original, el edificio continuaba presentando irregularidad en planta y en altura debido a las considerables distorsiones de piso y rotaciones que muestran grandes desplazamientos en la esquina de fachada del edificio. La situación es más acentuada para la dirección X como se puede apreciar en las imágenes, presentando en la dirección $Z$ un comportamiento prácticamente traslacional sin inconvenientes (Figura 4 y 5 ). 

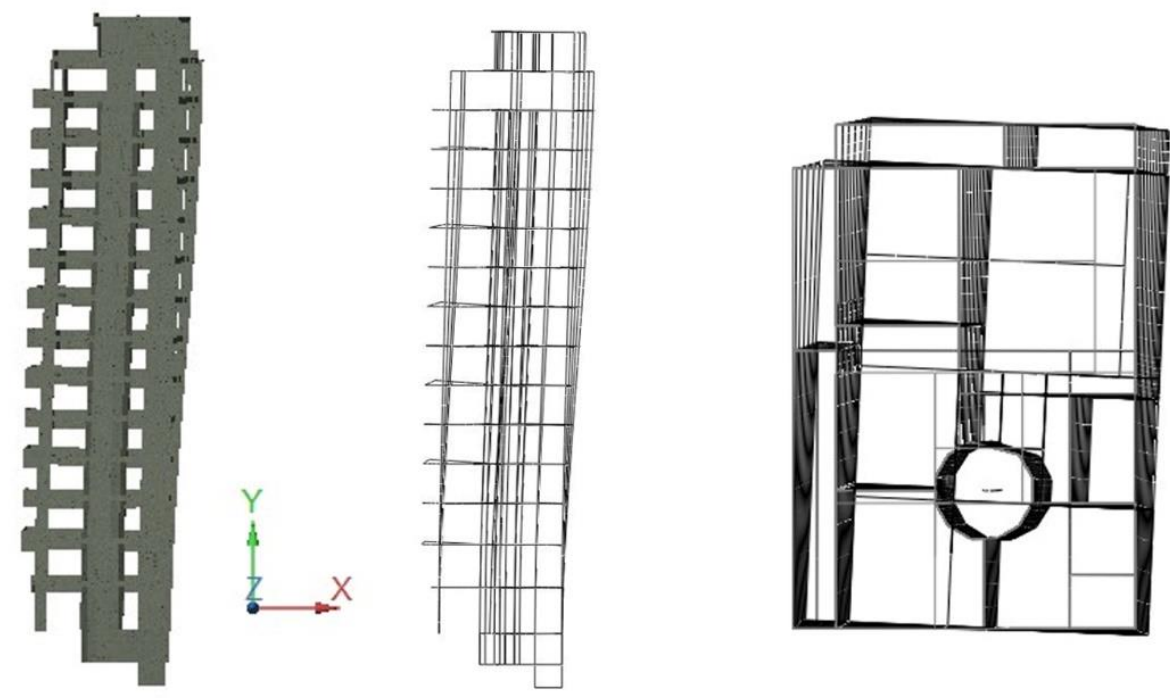

Figura 4. Deformada en vista y planta del edificio en dirección X.
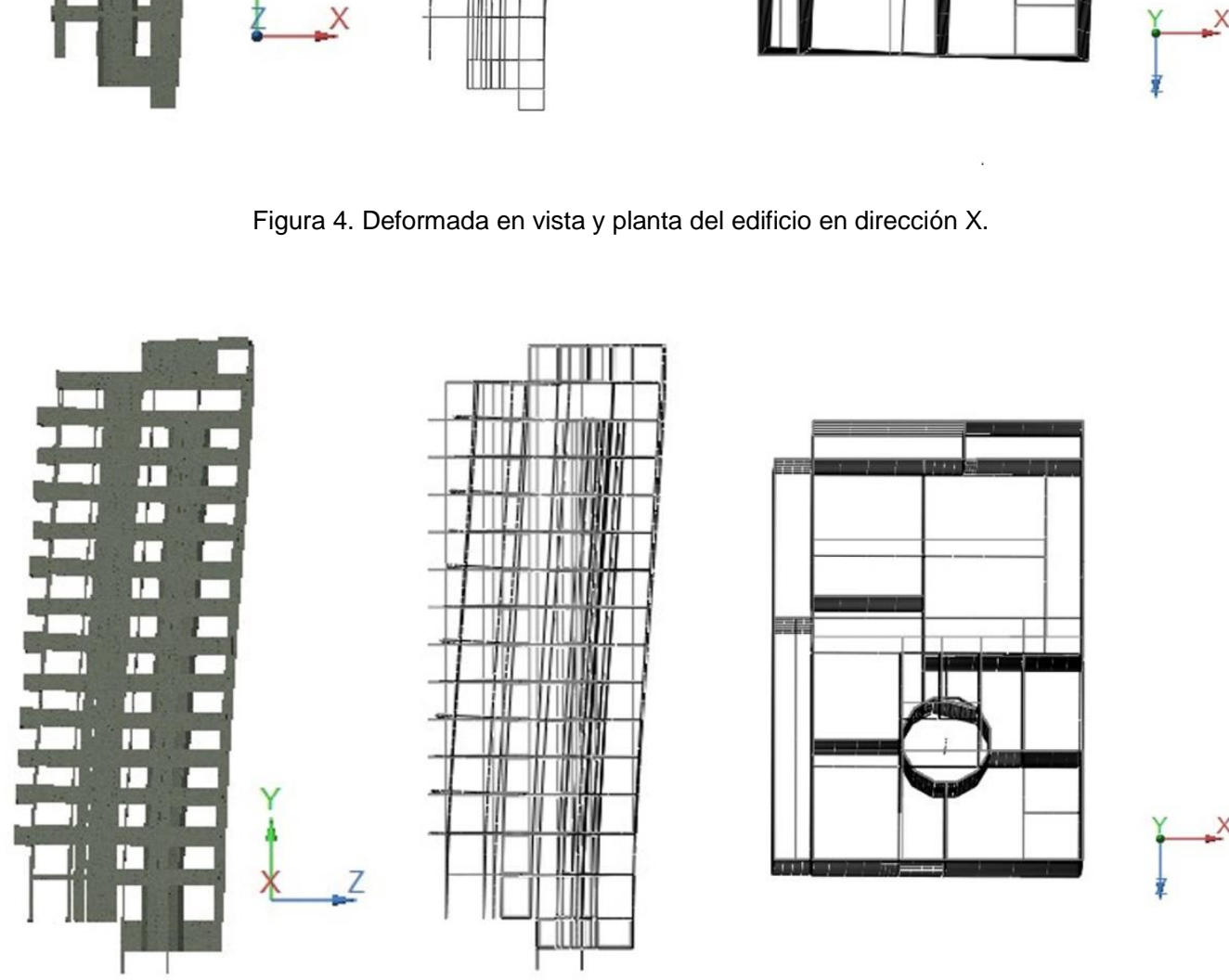

Figura 5. Deformada en vista y planta del edificio en dirección Z.

Para reducir estos efectos se procedió al aumento de rigidez en la esquina más deformable del edificio, aumentando el espesor de los tabiques existentes de tal forma de no invadir la ochava en la vereda ni modificar las premisas de accesibilidad del proyecto original (Figura 6). 

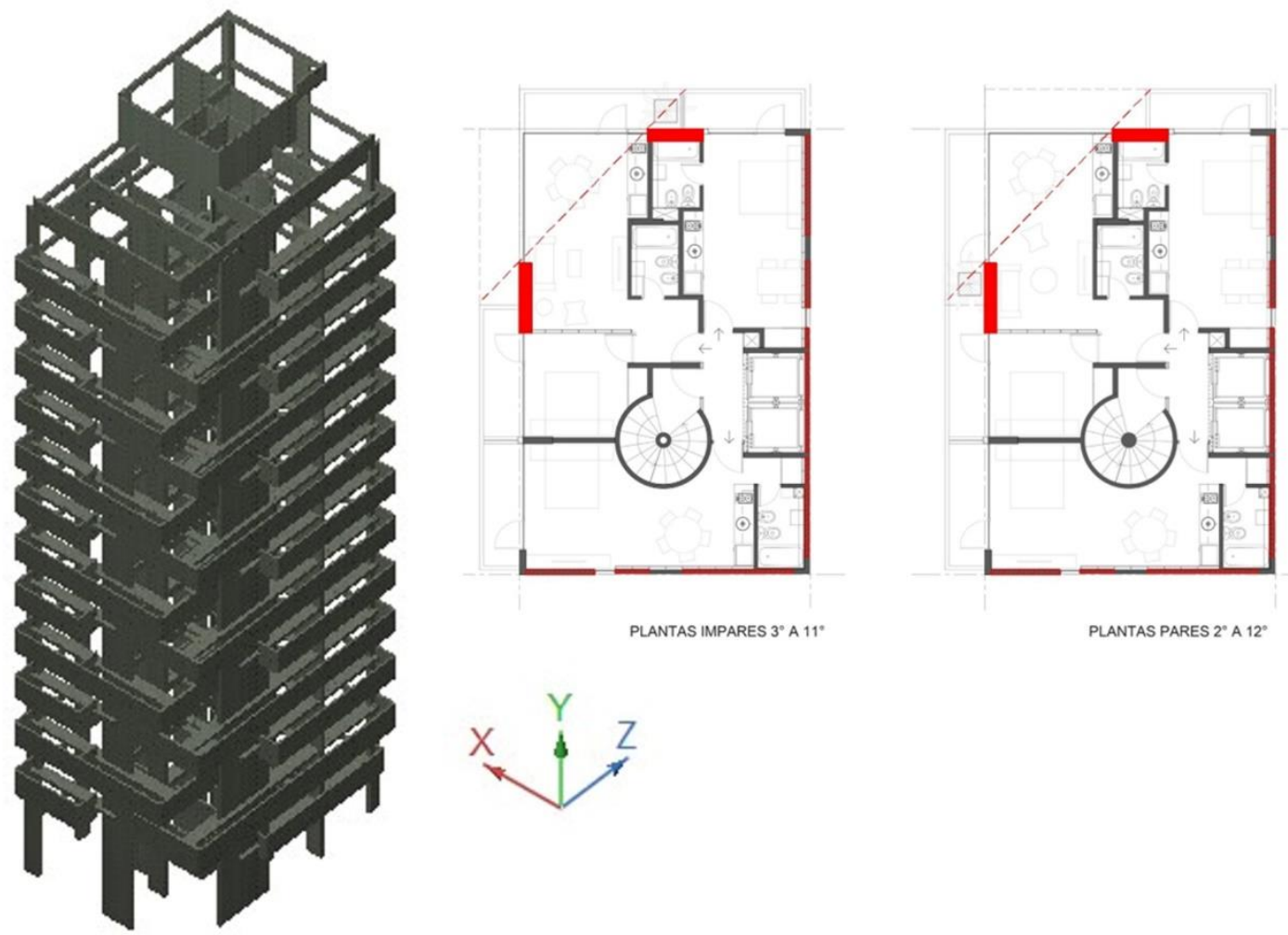

Figura 6. Modelado de propuesta estructural optimizada

A partir de las nuevas modificaciones se observa como las deformadas en ambas direcciones presentan comportamientos traslacionales con valores de regularidad en planta que corresponden a una estructura de irregularidad media y baja. La deformada en vertical no presenta grandes distorsiones ni desplazamientos localizados. Ambas condiciones nos garantizan un comportamiento más predecible del edificio sometido a la acción sísmica (Figuras 7 y 8$)$.
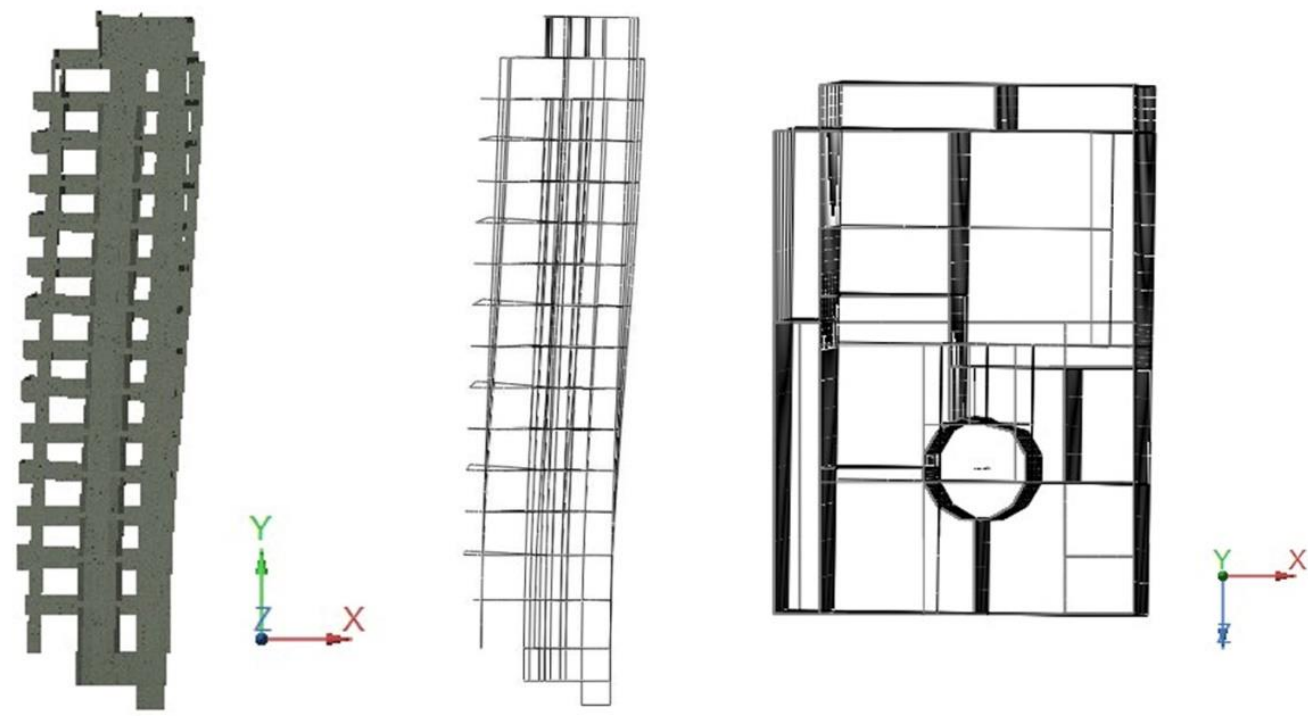

Figura 7. Deformada en vista y planta del edificio en dirección X. 

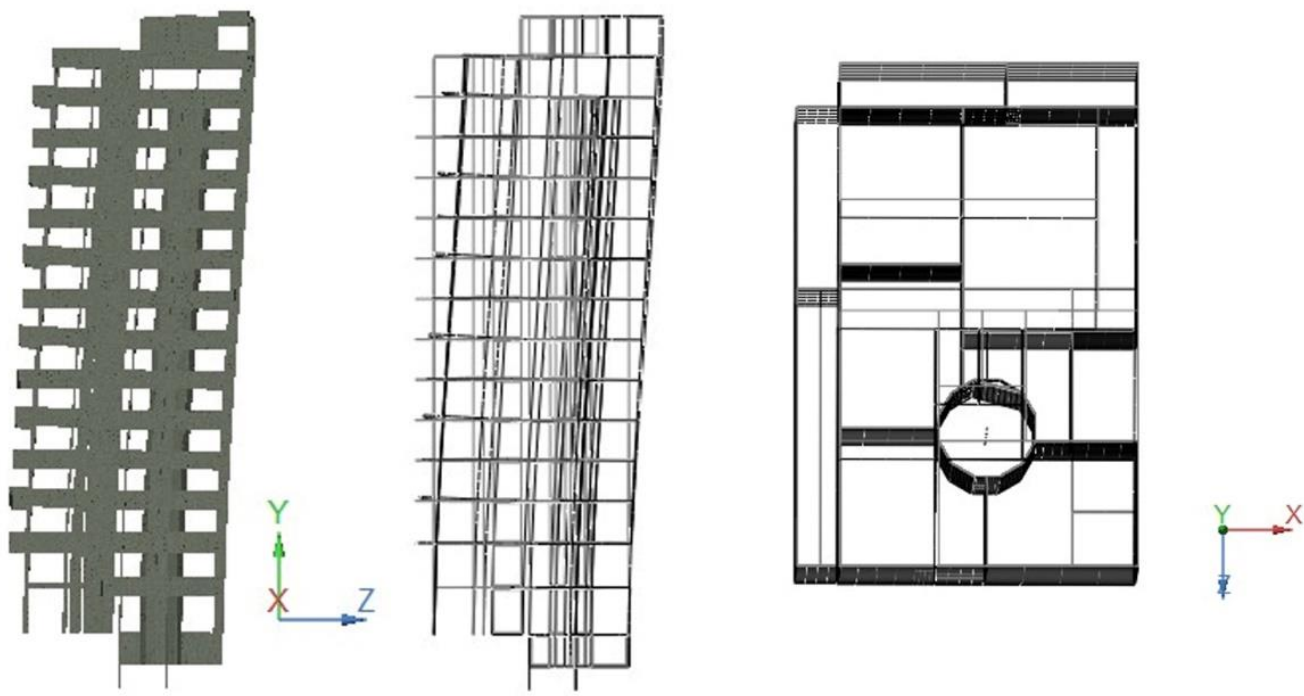

Figura 8. Deformada en vista y planta del edificio en dirección Z.

De este análisis se pudo constatar que el diseño estructural resultante resolvió todos los inconvenientes detectados, tanto en el proyecto original como en la primera alternativa analizada, enmarcándolo dentro de los parámetros de en un diseño regular según el INPRES-CIRSOC 103.

\section{Etapa 4}

Luego de evaluar todas las alternativas los primeros resultados arrojaron los siguientes valores:

- Proyecto original: el proyecto original, atendiendo a demandas estructurales y estéticas, contradicen los requerimientos para que una estructura sea considerada regular según los reglamentos, ineludibles para zonas de sismicidad media y elevada, demanda un consumo de 888 toneladas de hormigón armado para todos sus elementos estructurales. Esto equivaldría aproximadamente a 370 metros cúbicos de material sin haber analizado hasta el momento las cuantías requeridas para los elementos estructurales de cada alternativa.

- Primera alternativa: para esta alternativa el consumo de material requerido fue de 744 toneladas de hormigón armado o 310 metros cúbicos. Aunque la reducción del material fue considerable esta alternativa no respondía a los estándares de seguridad que se buscaban en esta investigación, por lo tanto, fue descartada.

- Segunda alternativa: finalmente en esta alternativa se logró reducir todos los errores detectados previamente ajustándonos a un diseño estructural regular logrando un consumo de hormigón armado de 777 toneladas o 324 metros cúbicos. Si bien no se evaluaron las cuantías requeridas por el momento sí es posible advertir que, al disminuirse considerablemente las rotaciones en planta del edificio, los elementos estructurales más demandados tendrán un comportamiento mucho más eficiente frente a la misma demanda de acciones gravitatorias, resultando menores cuantías y por lo tanto mayor facilidad constructiva.

\section{CONCLUSIONES}

Si bien aún se continúa desarrollando la etapa 4 del proyecto, el procedimiento llevado a cabo y sus resultados nos permitió elaborar estas primeras conclusiones:

$\rightarrow$ Una estructura regular además de tener un comportamiento más previsible y seguro durante los terremotos garantizan un menor consumo de los recursos materiales. 
$\rightarrow$ Si bien en la modernidad la eficiencia solo relacionaba consumo de materiales con demandas de solicitaciones, según los estándares actuales este requisito debiera estar atravesado por la seguridad estructural. No se puede hablar de eficiencia sin incluir la seguridad.

$\rightarrow$ Para las estructuras, un diseño regular constituye un recurso para protección pasivo y económico frente a los terremotos. La arquitectura para la vivienda debiera constituir un refugio para sus ocupantes.

$\rightarrow$ Una estructura regular y segura posee mayor durabilidad pudiendo ampliar su ciclo de vida al admitir futuras intervenciones.

$\rightarrow$ El diseño conceptual de una estructura sigue siendo la principal herramienta de diseño de un arquitecto por encima de los programas de cálculo estructural por más sofisticados que estos sean.

$\rightarrow$ Los arquitectos y futuros arquitectos debieran contar con instrumentos adaptados a los nuevos estándares normativos que les permitan incorporar desde las primeras ideas de partido, alternativas certeras para un diseño estructural sostenible y seguro.

En la actualidad el equipo se encuentra abocado a la elaboración de material didáctico que permita la transferencia de las conclusiones a las prácticas académicas.

Además, el camino transitado nos abrió las puertas a nuevos interrogantes como: ¿qué relación existe entre la esbeltez de un edificio y regularidad en altura? ¿qué rol cumplen las arquitecturas blandas para garantizar la regularidad estructural en zonas sísmicas elevadas? ¿qué rol tiene un buen diseño estructural en el grado de sostenibilidad de arquitecturas intervenidas? Estos cuestionamientos darán espacio a futuras investigaciones.

\section{BIBLIOGRAFÍA}

Barrios Corpa, J. y Santos Olalla, F. (2012) El proyecto de estructura en los sistemas de certificación sostenible de edificios. Málaga: $7^{\circ}$ congreso Aula Greencities.

Fernández, L; Gutiérrez, M. (2013) Bienestar Social, Económico y Ambiental para las Presentes y Futuras Generaciones. Información Tecnológica Vol. 24 (2), 121-130. ISSN 0718-0764

Fraga, J. M. (2017) Nuevo modelo de evaluación de la sostenibilidad de estructuras de hormigón (Tesis de doctorado) Universidad da Coruña. Recuperado de https://ruc.udc.es/dspace/handle/2183/19931.

IHOBE, sociedad pública de gestión ambiental, Editor (2010) Green building rating sistems: ¿Cómo evaluar la sostenibilidad en la edificación? Recuperado de https://www.ihobe.eus/publicaciones/green-building-rating-systems-como-evaluarsostenibilidad-en-edificacion

INPRES CIRSOC 103 (2018) Reglamento Argentino para construcciones sismorresistentes. Parte I: Construcciones en general. Reglamento y comentarios. Recuperado de http://www.inti.gob.ar/cirsoc/

Prados, S. (2018). Uso del hormigón armado en edificios de vivienda de la última década en Argentina y su relación con la confiabilidad estructural (Trabajo final de Especialidad) Córdoba: FAUDUNC. Recuperado de https://rdu.unc.edu.ar/handle/11086/8899

Prados, S.; Cardellino, A. (2020). Diseño estructural sostenible en Hormigón Armado. Libro: Ambiente, tecnología y diseño sustentable: las preexistencias ambientales y su impacto en la calidad de vida, el confort y la eficiencia energética. Cap. 9 (p. 118-129) Córdoba: FAUD-UNC.

Prados, S.; Cardellino, A. (2020). Diseño estructural sostenible en Hormigón Armado. Libro: VIII Jornadas de Investigación y II Jornadas de Investigación de Becarios y Doctorandos Encuentro y Reflexión: investigación + transferencia + desarrollo. (p. 133-141). Córdoba: FAUD-UNC. Recuperado de https://rdu.unc.edu.ar/handle/11086/15566

Sgrelli, E. (2017). Hormigón sostenible en Argentina. Estado del arte y buenas prácticas. Ciudad Autónoma de Buenos Aires, Consejo Profesional de Ingeniería Civil. ISBN: 978-987-95422-79 